\title{
Expression of miR-34a and Ki67 in nasopharyngeal carcinoma and the relationship with clinicopathological features and prognosis
}

\author{
LINQI WEI*, CHAO SHI ${ }^{*}$ and YONGHONG ZHANG \\ Department of Otolaryngology, Head and Neck Surgery, Xiangyang No. 1 People's Hospital, \\ Hubei University of Medicine, Xiangyang, Hubei 441000, P.R. China
}

Received June 5, 2019; Accepted October 22, 2019

DOI: $10.3892 / 01.2019 .11217$

\begin{abstract}
Expression levels of miR-34a and Ki67 in nasopharyngeal carcinoma (NPC) and the relationship with clinicopathological features and prognosis were studied. A prospective study was performed on 56 cases of NPC tissues and 56 cases of adjacent tissues collected in Xiangyang No. 1 People's Hospital. The expression levels of miR-34a, Ki67 in NPC and adjacent tissues were detected by RT-qPCR. The association among the expression levels of miR-34a and Ki67, the clinicopathological features and prognosis of patients was analyzed. The relative expression levels of miR-34a in 56 cases of NPC were lower than those of the adjacent tissues. The expression of miR-34a in NPC was significantly associated with bone metastasis and TNM staging $(\mathrm{P}<0.001)$. The relative expression of Ki67 in 56 cases of NPC was higher than that of the adjacent tissues. The expression of Ki67 in NPC was significantly associated with lymphatic metastasis and TNM staging $(\mathrm{P}<0.001)$. The 5-year survival of patients with low expression of miR-34a was significantly lower than that of patients with high expression, and the survival of patients with high expression of Ki67 was significantly lower than that of patients with low expression. According to Pearson's correlation analysis, Ki67 expression was negatively correlated with miR-34a expression in NPC tissues. In conclusion, the expression of Ki67 in NPC was upregulated, while the expression of miR-34a in NPC was downregulated. miR-34a expression in NPC was significantly associated with bone metastasis and TNM staging, and Ki67 expression in NPC was significantly
\end{abstract}

Correspondence to: Dr Yonghong Zhang, Department of Otolaryngology, Head and Neck Surgery, Xiangyang No. 1 People's Hospital, Hubei University of Medicine, 15 Jiefang Road, Xiangyang, Hubei 441000, P.R. China

E-mail: ywun98@163.com; 940155243@qq.com

\section{*Contributed equally}

Key words: miR-34a, Ki67, nasopharyngeal carcinoma, clinicopathological features associated with lymphatic metastasis and TNM staging. In addition, there was a negative correlation between miR-34a and Ki67 expression levels, and the two can be used as predictors of NPC-associated mortality. The expression levels of miR-34a and Ki67, as well as TNM staging were associated with the prognosis of NPC patients.

\section{Introduction}

Nasopharyngeal carcinoma (NPC) is a head and neck cancer with low survival rate and a fairly high incidence in its endemic region (1). The reported incidence of NPC in China is relatively higher than that in other parts of the world, with an incidence of $3.26 / 100,000$. The incidence of NPC in males is $4.51 / 100,000$, which is 2.32 times higher than that in females, and the mortality rate in China is $1.77 / 100,000(2,3)$. The incidence of NPC in China is also regional; the incidence of NPC in the southern region is as high as 50/100,000 (4). At present, the main treatment for NPC is radiotherapy, chemotherapy, and their combination. However, the survival rate after treatment tends to decrease with the development of the disease. The 10 -year overall survival rates for stages I, II, III and IV are 100, 87.1, 75.5 and $55.6 \%$, respectively $(5,6)$. The main causes of poor prognosis in NPC patients are local treatment failure and distant metastasis (7). Therefore, finding prognostic factors for NPC is more conducive compared to timely optimal treatment in improving the disease outcome.

MicroRNA, a small single stranded RNA molecule, has been widely studied. Some microRNAs have been found to be related to the development and disease in the process of life (8). The effect of microRNAs on NPC has also been studied. Cheng et al (9) have shown that the low expression of miR-122 can inhibit the proliferation of NPC cells and promote their apoptosis. Chen et al (10) have shown that miR-203a-3p inhibits the growth and metastasis of NPC tumors. In recent years, studies on miR-34a have been reported and have shown that miR-34a can inhibit the proliferation and metastasis of oral squamous carcinoma cells (11). However, whether miR-34a expression is also associated with NPC has not been clarified yet.

Ki67 is a marker used to evaluate cell proliferation and is highly expressed in malignant cells. However, Ki67 is often 
undetectable in normal cells. Therefore, it is often used to predict the progression of cancer (12). It has been reported that Ki67 can be used as an independent prognostic indicator of NPC, and its increase is associated with poor prognosis (13). However, there are few studies on the relationship between the clinicopathological features of NPC and Ki67.

In the present study, we investigated the expression levels of miR-34a and Ki67 in NPC, and explored their relationship with clinicopathological features and prognosis, to provide a reference for clinical use.

\section{Patients and methods}

Clinical data of patients. A prospective study was performed on cancer tissues of 56 NPC patients who underwent needle biopsy from March 2011 to December 2013 in Xiangyang No. 1 People's Hospital (Xiangyang, China). These tissues were selected as the study group, while the adjacent tissues $(\sim 2 \mathrm{~cm}$ away from the lesion) comprised the control group. There were 41 males and 15 females with an average age of $52.44 \pm 13.75$ years. The clinical staging criteria were based on the NPC Diagnostic Criteria (14), as set out by the Cancer Staging Guide of the American Joint Committee on Cancer in 2010. The study was approved by the Medical Ethics Committee of Xiangyang No. 1 People's Hospital (no. JS2011HHHC012) and all the patients were informed and signed an informed consent form.

Inclusion and exclusion criteria. Inclusion criteria: Patients diagnosed with NPC by imaging and pathology; patients that hadn't undergone radiotherapy and chemotherapy before the needle biopsy; patients without distant metastasis; patients with complete clinical data; patients that could be followed up by telephone. Exclusion criteria: Patients with other otorhinolaryngological diseases; patients with other malignant tumors; patients with severe cardio-cerebrovascular disease; patients with severe inflammation; pregnant or lactating women.

Main instruments and reagents. PCR instrument (ABI 7500; Applied Biosystems; Thermo Fisher Scientific, Inc.), Ultraviolet spectrophotometer (6135000041; Eppendorf, Inc.), total RNA extraction EasyPure miRNA kit (ER601-01; Beijing Transgen Biotech Co., Ltd.), reverse transcription and PCR kit TransScript miRNA First-Strand cDNA Synthesis SuperMix (AT351-01; Beijing Transgen Biotech Co., Ltd.). TransStart ${ }^{\circledR}$ Tip Green qPCR SuperMix (+Dye II) (AQ142-21; Beijing Transgen Biotech Co., Ltd.). All primers were designed and synthesized by Shanghai Shenggong Biological Co., Ltd. (Table I).

$R T$ - $q P C R$ detection method. Tissue $(3 \mathrm{~mm})$ stored at $-80^{\circ} \mathrm{C}$ was ground in liquid nitrogen. Total RNA from tissue suspension was extracted in strict accordance with the manufacturer's instructions of total RNA extraction kit. The purity and concentration of RNA were detected using ultraviolet spectrophotometer and agarose gel electrophoresis. Total RNA was then reversely transcribed using the TransScript ${ }^{\circledR}$ miRNA RT Enzyme Mix and 2X TS miRNA Reaction Mix (AT351-01; Beijing Transgen Biotech Co., Ltd.). After shaking, total RNA was insulated at $30^{\circ} \mathrm{C}$ for $5 \mathrm{~min}$ and then incubated at $42^{\circ} \mathrm{C}$ for $50 \mathrm{~min}$ in strict accordance with the manufacturer's instructions. Next, PCR amplification was carried out in a $20-\mu 1$ reaction volume containing $1 \mu \mathrm{l}$ of
cDNA, $0.4 \mu \mathrm{l}$ of each upstream and downstream primers, $10 \mu \mathrm{l}$ of 2X TransStart ${ }^{\circledR}$ Tip Green qPCR SuperMix and $0.4 \mu \mathrm{l}$ of Passive Reference Dye (50X), to a final volume made up by $\mathrm{ddH}_{2} \mathrm{O}$. PCR conditions were as follows: Pre-denaturation at $94^{\circ} \mathrm{C}$ for $30 \mathrm{sec}$, denaturation at $94^{\circ} \mathrm{C}$ for $5 \mathrm{sec}$, and annealing and extension at $60^{\circ} \mathrm{C}$ for $30 \mathrm{sec}$, for a total of 40 cycles. Each sample was tested in 3 repeat wells, and the experiment was carried out 3 times. U6 and GAPDH were used as internal reference for miR-34a and $\mathrm{Ki67}$, respectively, and the $2^{-\Delta \Delta \mathrm{Cq}}$ method (15) was used to analyze the data.

Follow up. Fifty-six patients were followed up by telephone and visit. Follow-up was conducted every 3 months for 1-2 years after operation, and then at each 6 months for 2-5 years after operation. The follow-up period was 5 years and the deadline was December 2018. All patients were followed up, with an average time of 51 months. The overall survival time was from the first day of the operation to the last follow-up or death.

Outcome measures. The expression levels of miR-34a and Ki67 in the study and the control group were compared, and the 5-year survival of the patients was recorded. At 5 years, the patients were divided into two groups: The survival group and the deceased group. Multivariate COX regression was used to analyze the relevant factors and Pearson's correlation analysis was used to analyze the correlation between miR-34a and Ki67 in NPC tissues.

The optimal cut-off values of miR-34a- and Ki67-associated mortality in NPC were analyzed by receiver operating characteristic (ROC) curves. The best cut-off values of miR-34a and Ki67 were divided into high- and low-expression groups to observe the 5-year mortality and to draw Kaplan-Meier survival curves.

Statistical analysis. SPSS 20.0 medical statistical analysis software (IBM Corp.) was used for the statistical analysis of the collected data, and GraphPad Prism 7 (GraphPad Software, Inc.) for plotting the figures. Enumeration data were expressed as rate (\%) and tested by Chi-square test. Measurement data were expressed as the mean \pm standard deviation (means \pm SD). All measurement data followed a normal distribution. Independent samples t-test was used for their comparison between two groups. The 5-year survival was analyzed by Kaplan-Meier survival analysis. The ability of miR-34a and Ki67 to diagnose NPC-associated mortality was analyzed by log-rank test and was evaluated by ROC curves analysis. Multiple factor Cox regression test was used for multivariate analysis of survival. Pearson's correlation analysis was used for analyzing the correlation between miR-34a and Ki67 expression in NPC tissues. $\mathrm{P}<0.05$ was considered to indicate a statistically significant difference.

\section{Results}

Expression of miR-34a and Ki67 in the study and control groups. By comparing the expression levels of miR-34a and Ki67 between the two groups, it was found that the relative expression of miR-34a in the study group was significantly lower than that in the control group, and there was a significant difference between the two groups $(\mathrm{P}<0.05)$. The relative 
Table I. Primer sequences.

\begin{tabular}{lll}
\hline Genes & \multicolumn{1}{c}{ Upstream primers } & \multicolumn{1}{c}{ Downstream primers } \\
\hline miR-34a & 5'-CTAGCTAGCTTGCTGTCCGCTTGATACTGG-3' & 5'-GGAAGATCTCCCCGATCTGGTCACCGA-3' \\
Ki67 & 5'-AAACCCCACCAAGTAAAACA-3' & 5'-CCAAGGCAAGCTCAGGAC-3' \\
GAPDH & 5'-AAAGAAGCTCAACTACATGG-3' & 5'-TGCAAAGAATGCGTCCCAGAG-3' \\
U6 & 5'-CTCGCTTCGGCAGCACA-3' & 5'-AACGCTTCACGAATTTGCGT-3'
\end{tabular}

Table II. Expression levels of miR-34a and Ki67 in the study and control groups.

\begin{tabular}{|c|c|c|c|c|}
\hline Index & Study group $(n=56)$ & Control group $(n=56)$ & t value & P-value \\
\hline miR-34a & $4.285 \pm 0.208$ & $5.147 \pm 0.257$ & 19.510 & $<0.001$ \\
\hline Ki67 & $8.673 \pm 0.435$ & $6.947 \pm 0.237$ & 26.073 & $<0.001$ \\
\hline
\end{tabular}

Table III. Association of miR-34a and Ki67 with the clinicopathological features in patients with NPC.

\begin{tabular}{|c|c|c|c|c|c|c|c|}
\hline Clinicopathological features & $\mathrm{n}$ & $\operatorname{miR}-34 a(n=56)$ & $\mathrm{t}$ value & P-value & Ki67 $(n=56)$ & t value & P-value \\
\hline Age (years) & & & 0.302 & 0.764 & & 0.196 & 0.846 \\
\hline$<50$ & 27 & $4.804 \pm 0.212$ & & & $8.037 \pm 0.368$ & & \\
\hline$\geq 50$ & 29 & $4.787 \pm 0.209$ & & & $8.018 \pm 0.359$ & & \\
\hline Sex & & & 0.462 & 0.646 & & 0.662 & 0.511 \\
\hline Male & 41 & $4.675 \pm 0.221$ & & & $7.856 \pm 0.372$ & & \\
\hline Female & 15 & $4.706 \pm 0.226$ & & & $7.784 \pm 0.326$ & & \\
\hline Smoking history & & & 1.627 & 0.110 & & 0.063 & 0.950 \\
\hline Yes & 25 & $4.497 \pm 0.217$ & & & $8.022 \pm 0.418$ & & \\
\hline No & 31 & $4.616 \pm 0.224$ & & & $8.015 \pm 0.415$ & & \\
\hline Differentiation & & & 0.898 & 0.373 & & 0.171 & 0.866 \\
\hline Undifferentiated & 14 & $4.772 \pm 0.236$ & & & $7.982 \pm 0.387$ & & \\
\hline Low and moderately differentiated & 42 & $4.844 \pm 0.267$ & & & $8.003 \pm 0.403$ & & \\
\hline Bone metastasis & & & 9.020 & $<0.001$ & & 0.069 & 0.945 \\
\hline Yes & 19 & $4.334 \pm 0.202$ & & & $8.029 \pm 0.417$ & & \\
\hline No & 37 & $4.947 \pm 0.258$ & & & $8.021 \pm 0.408$ & & \\
\hline Lymphatic metastasis & & & 0.708 & 0.482 & & 8.951 & $<0.001$ \\
\hline Yes & 18 & $4.578 \pm 0.225$ & & & $7.847 \pm 0.384$ & & \\
\hline No & 38 & $4.624 \pm 0.228$ & & & $6.984 \pm 0.313$ & & \\
\hline Local invasion of tumor & & & 0.725 & 0.472 & & 0.926 & 0.359 \\
\hline Yes & 24 & $4.734 \pm 0.243$ & & & $7.815 \pm 0.357$ & & \\
\hline No & 32 & $4.782 \pm 0.247$ & & & $7.729 \pm 0.334$ & & \\
\hline TNM staging & & & 10.110 & $<0.001$ & & 20.535 & $<0.001$ \\
\hline Stage I-II & 33 & $4.884 \pm 0.236$ & & & $6.324 \pm 0.305$ & & \\
\hline Stage III-IV & 23 & $4.263 \pm 0.211$ & & & $8.293 \pm 0.413$ & & \\
\hline
\end{tabular}

NPC, nasopharyngeal carcinoma.

expression of Ki67 in the study group was significantly higher than that in the control group $(\mathrm{P}<0.05)$ (Table II).

Association of miR-34a, Ki67 and clinicopathological features in patients with NPC. By analyzing the expression levels of miR-34a and Ki67, it was found that the expression of miR-34a was significantly associated with bone metastasis and TNM staging $(\mathrm{P}<0.05)$. The expression of Ki67 in NPC was significantly associated with lymphatic metastasis and TNM staging $(\mathrm{P}<0.05)$ (Table III). 
Table IV. Diagnostic value of miR-34a, Ki67, and their combination in NPC-associated mortality.

\begin{tabular}{lcccrrr}
\hline Index & AUC & $95 \%$ CI & Specificity $(\%)$ & Sensitivity $(\%)$ & Youden index (\%) & Cut-off \\
\hline miR-34a & 0.785 & $0.649-0.922$ & 68.29 & 80.00 & 48.29 & $<4.680$ \\
Ki67 & 0.772 & $0.616-0.929$ & 87.80 & 60.00 & 47.80 & $>8.073$ \\
Joint detection & 0.855 & $0.734-0.977$ & 82.93 & 73.33 & 52.68 & $>0.271$ \\
\hline
\end{tabular}

NPC, nasopharyngeal carcinoma; AUC, area under curve.

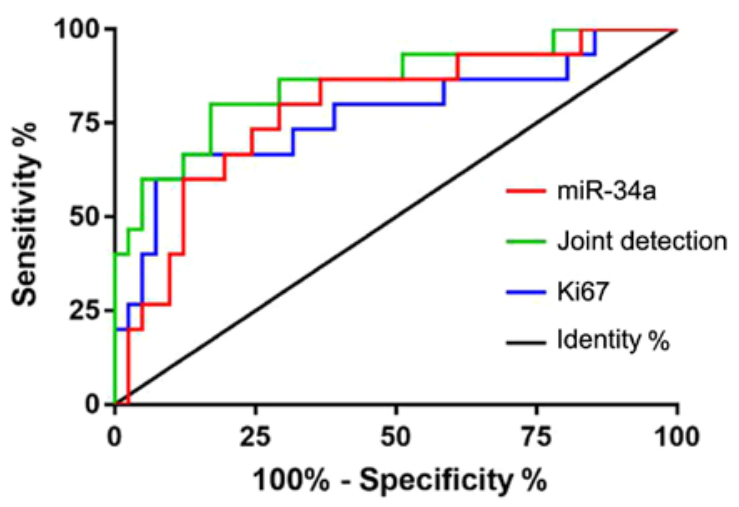

Figure 1. ROC curves for predicting NPC mortality. ROC, receiver operating characteristic; NPC, nasopharyngeal carcinoma.

Diagnostic value of miR-34a and Ki67 in NPC-associated mortality. Patients were divided into survival group and deceased group according to their survival. ROC curves were drawn for the expression levels of miR-34a and $\mathrm{Ki} 67$ in both groups to analyze the diagnostic value of the two indexes in NPC. The area under curve (AUC) for miR-34a was 0.785 , the $95 \%$ CI was $0.649-0.922$, the specificity was $68.29 \%$, the sensitivity was $80.00 \%$, and the cut-off value was 4.680 . The AUC of Ki67 was 0.772 , the $95 \%$ CI was $0.616-0.929$, the specificity was $87.80 \%$, the sensitivity was $60.00 \%$, and the cut-off value was 8.073. The AUC of the joint detection was 0.855 , the $95 \%$ CI was $0.734-0.977$, the specificity was $82.93 \%$, the sensitivity was $73.33 \%$, and the cut-off value was 0.271 (Table IV and Fig. 1).

Relationship of the expression levels of miR-34a and Ki67 with the 5-year survival of patients. According to the 5-year survival analysis of patients, 56 cases were followed up and no case was lost. All patients were followed up; 15 patients died and 41 survived within 5 years, and the survival rate was $73.21 \%$. According to the median value of miR-34a and Ki67 expression levels, the patients were divided into high- and low-expression groups. The Kaplan-Meier survival analysis revealed that the survival rate of patients with low expression of miR-34a was significantly lower than that of patients with high miR-34a expression, and there was significant difference between the two groups $(\mathrm{P}<0.001)$. According to the observation of $\mathrm{Ki} 67$ expression in two groups of patients, it was found that the survival of patients with high expression of Ki67 was significantly lower than that of patients with low expression, and there was a significant difference between the two groups $(\mathrm{P}=0.022)$ (Fig. 2).

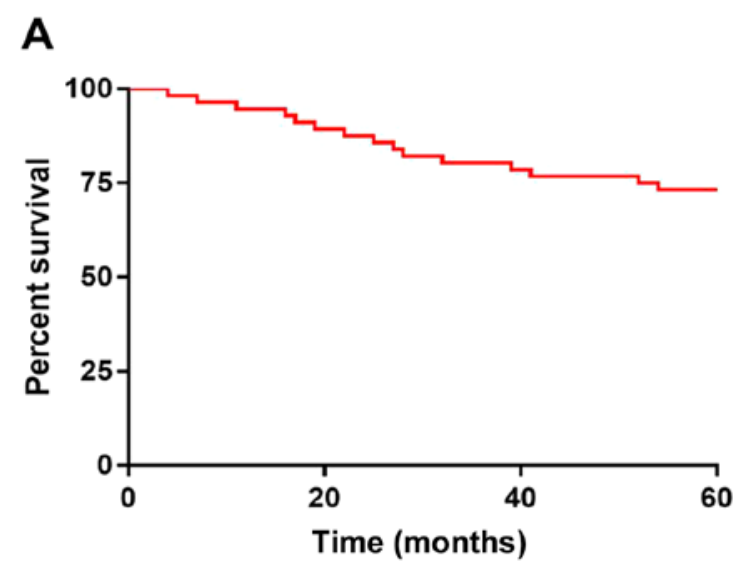

B
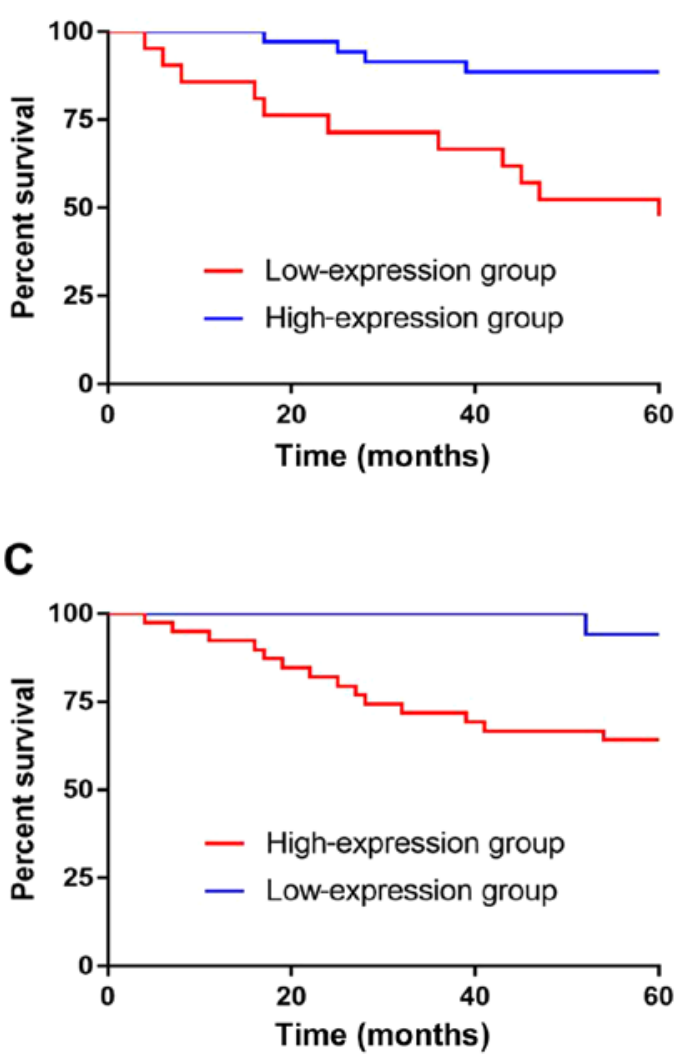

Figure 2. miR-34a and Ki67 expression levels and the 5-year survival of patients. (A) The 5-year follow-up showed that the overall survival rate was $73.21 \%$. (B) The survival rate of patients with low expression of miR-34a was significantly lower than that of patients with high miR-34a expression, and there was a significant difference between the two groups $(\mathrm{P}<0.001)$. (C) According to the observation of Ki67 expression in the two groups of patients, the survival rate of patients with high expression of Ki67 was significantly lower than that of patients with low Ki67 expression, and there was a significant difference between the two groups $(\mathrm{P}=0.022)$. 
Table V. Univariate analysis.

\begin{tabular}{|c|c|c|c|c|}
\hline Factors & Survival group $(\mathrm{n}=41)$ & Deceased group $(n=15)$ & $\mathrm{t} / \chi^{2}$ value & P-value \\
\hline Age (years) & & & 0.020 & 0.889 \\
\hline$<50$ & $20(48.78)$ & $7(46.67)$ & & \\
\hline$\geq 50$ & $21(51.22)$ & $8(53.33)$ & & \\
\hline Sex & & & 0.448 & 0.503 \\
\hline Male & $31(75.61)$ & $10(66.67)$ & & \\
\hline Female & $10(24.39)$ & $5(33.33)$ & & \\
\hline Smoking history & & & 0.034 & 0.854 \\
\hline Yes & $18(43.90)$ & $7(46.67)$ & & \\
\hline No & $23(56.10)$ & $8(53.33)$ & & \\
\hline Differentiation & & & 5.130 & 0.024 \\
\hline Undifferentiated & $7(17.07)$ & $7(46.67)$ & & \\
\hline Low and moderately differentiated & $34(82.93)$ & $8(53.33)$ & & \\
\hline Bone metastasis & & & 6.212 & 0.013 \\
\hline Yes & $10(24.39)$ & $9(60.00)$ & & \\
\hline No & $31(75.61)$ & $6(40.00)$ & & \\
\hline Lymphatic metastasis & & & 2.574 & 0.040 \\
\hline Yes & $10(24.39)$ & $8(53.33)$ & & \\
\hline No & $31(75.61)$ & $7(46.67)$ & & \\
\hline Local invasion of tumor & & & 4.743 & 0.029 \\
\hline Yes & $14(34.15)$ & $10(66.67)$ & & \\
\hline No & $27(65.85)$ & $5(33.33)$ & & \\
\hline TNM staging & & & 17.598 & $<0.001$ \\
\hline Stage I-II & $31(75.61)$ & $2(13.33)$ & & \\
\hline Stage III-IV & $10(24.39)$ & $13(86.67)$ & & \\
\hline miR-34a & $4.942 \pm 0.247$ & $4.221 \pm 0.212$ & 10.022 & $<0.001$ \\
\hline Ki67 & $7.643 \pm 0.352$ & $8.547 \pm 0.427$ & 8.034 & $<0.001$ \\
\hline
\end{tabular}

Univariate analysis of patient survival. The clinical data of patients in the survival and deceased group were collected and analyzed by univariate analysis. The results showed that there was no significant difference in age, sex and smoking history between the two groups $(\mathrm{P}>0.05)$. However, there were significant differences in differentiation degree, bone metastasis, lymphatic metastasis, local invasion of tumor, TNM staging, miR-34a and Ki67 between the two groups $(\mathrm{P}<0.05)($ Table V).

Multivariate analysis of patient survival. Indices that differed from univariate analysis were incorporated into assignments (Table VI). The results of multivariate Cox regression analysis showed that the degree of differentiation, bone metastasis, lymphatic metastasis and local invasion of tumor were not independent factors for the survival of the patients. TNM staging (OR: $2.561,95 \%$ CI: 0.182-8.598), miR-34a (OR: 0.011, 95\% CI: 0.005-0.681) and Ki67 (OR: 0.016, 95\% CI: 0.002-2.278) were independent factors in NPC patients (Table VII).

Correlation analysis of miR-34a and Ki67 expression levels in $N P C$. There was a negative correlation between the expression levels of miR-34a and Ki67 in NPC tissues, as predicted by
Table VI. Assignments.

Factors

Assignment

Degree of differentiation

Undifferentiated $=1$, Lowly and moderately differentiated $=0$

Bone metastasis Yes $=1, \mathrm{No}=0$

Lymphatic metastasis Yes $=1, \mathrm{No}=0$

Local invasion of tumor TNM staging miR-34a Yes $=1, \mathrm{No}=0$ Stage III $+\mathrm{IV}=1$, Stage $\mathrm{I}+\mathrm{II}=0$ Data belong to continuous variables using raw data analysis Ki67 Data belong to continuous variables using raw data analysis

Survival time Data belong to continuous variables using raw data analysis Survival status

Pearson's correlation analysis. The expression of Ki67 was found to decrease with the increase of miR-3a expression $(\mathrm{r}=-0.502, \mathrm{P}<0.001)($ Fig. 3$)$. 
Table VII. Multivariate analysis.

$\operatorname{Exp}(\mathrm{B}) 95 \% \mathrm{CI}$

\begin{tabular}{lrcccccc}
\cline { 6 - 8 } Factors & B & S.E. & Wals & Sig. & Exp (B) & Lower limit & Upper limit \\
\hline TNM staging & -7.925 & 3.848 & 4.242 & 0.039 & 2.561 & 0.182 & 8.598 \\
miR-34a & -13.839 & 5.838 & 5.620 & 0.018 & 0.011 & 0.005 & 0.681 \\
Ki67 & 4.262 & 2.140 & 3.966 & 0.030 & 0.016 & 0.002 & 2.278 \\
\hline
\end{tabular}

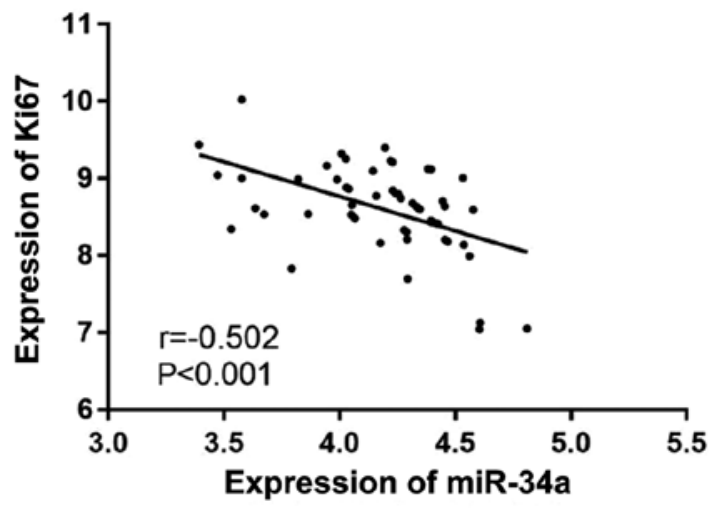

Figure 3. Correlation analysis of miR-34a and Ki67 expression levels. There was a negative correlation between the expression levels of miR-34a and Ki67 ( $r=-0.502, \mathrm{P}<0.001)$.

\section{Discussion}

NPC is a common malignant tumor in South China and Southeast Asia (16). The specific etiology of NPC is still unclear, however, it has been reported that NPC is related to EBV virus infection, and methylation of the parkin gene may also promote tumors of NPC $(17,18)$. Although NPC patients are sensitive to radiotherapy, the prognosis of NPC patients is still poor due to recurrence and distant metastasis (19). Therefore, finding biomarkers that affect the prognosis of NPC is of great significance to improve the prognosis and survival rate of the patients.

In recent years, there has been a series of studies on microRNAs in NPC. In the study of Sanchez Calle et al (20), a series of biological assays were carried out on NPC cell line in vitro and in vivo, and it was found that miR-130a-3p was downregulated in both NPC tissue and cell line. This result is similar to the result of the present study, that the expression of miR-34a in cancer tissue samples is lower than that in adjacent tissues. Huang et al (21) found that miR-34a inhibited epithelial mesenchymal transformation, invasion and migration of NPC cells induced by TGF- $\beta$ through directly targeting Smad4. However, the relationship between miR-34a and clinicopathological features and prognosis of NPC has rarely been reported. Therefore, in the present study, the relationship between miR-34a and the clinicopathological features of the patients was further studied and it was found that the expression of miR-34a is significantly associated with bone metastasis and TNM staging. Ki67, used for the evaluation or as a prognostic indicator of breast cancer, gastrointestinal pancreatic neuroendocrine tumo, and other cancers, has been extensively studied in recent years $(22,23)$. In the present study, it was found that Ki67 expression in cancer tissues is significantly higher than that in adjacent tissues. The relationship between Ki67 and clinicopathological features revealed that the expression of Ki67 is significantly associated with lymphatic metastasis and TNM staging. Guan et al (24) detected the expression of Ki67 protein in 90 pairs of NPC and distant normal tissue by immunohistochemical method, and found that the expression of Ki67 in tumor was higher than that in normal tissue, which is similar to our results.

The 5-year survival of patients in this study was followed up. Of the total 56 patients, 15 patients died within 5 years, 41 patients survived, and the survival rate was $73.21 \%$. Patients were divided according to their survival, and the diagnostic value of miR-34a and Ki67 was analyzed by ROC curve analysis. The AUC of miR-34a was 0.785 , when the cut-off value was $<4.680$, and the optimum specificity and sensitivity were 68.29 and $80.00 \%$, respectively. The AUC for Ki67 was 0.772 , the optimum specificity and sensitivity were 87.80 and $60.00 \%$, respectively, when the cut-off value was $>8.073$. These results suggest that miR-34a and Ki67 may be used as predictors of NPC-associated mortality. At the same time, the sensitivity and specificity of the two indexes were significantly different from each other. Through the joint detection of the two indicators, it was found that the area under the joint detection curve was 0.855 , and the best specificity and sensitivity were 82.93 and $73.33 \%$, respectively, when the cut-off value was $>0.271$. These results suggest that the deficiency of the two can be overcome by joint detection. Patients were divided into high- and low-expression groups according to the median values of the two groups, and the Kaplan-Meier survival curves that were drawn. The 5-year survival of patients with low expression of miR-34a was significantly lower than that of patients with high miR-34a expression, and the 5-year survival of patients with high expression of Ki67 was significantly lower than that of patients with low Ki67 expression.

Zhao et al (25), reported on the 3-year overall survival and the high and low Ki67 expression in 45 patients with NPC. The results revealed that the survival rate of patients with high expression of Ki67 was lower, which is similar to our results. In another study, the high expression of Ki67 was considered to be beneficial to anti-radiation, leading to local recurrence and distant metastasis, which would result in poor prognosis (26). By analyzing the patients' clinicopathologic features, it was also found, in the present study, that the patients in stages I and II have lower expression of Ki67 and higher expression of miR-34a 
compared with those in stage III and IV. Thus, the proportion of patients in the early stage with low expression of Ki67 and high expression of miR-34a was higher, and their survival condition was better. Multivariate Cox regression analysis was also performed and it was found that TNM staging, miR-34a and Ki67 were independent factors for NCP-associated mortality, indicating that TNM staging, miR-34a and Ki67 could be used as clinical indexes to evaluate the prognosis of NPC patients. In addition, the correlation of miR-34a and Ki67 in NPC was detected by Pearson's correlation analysis, and it was found that there is a negative correlation between miR-34a and Ki67 expression levels, which suggests that there might be a close relationship between miR-34a and Ki67, although this was not explored in depth.

Although the relationship among miR-34a, Ki67, clinical pathology and prognosis of NPC was determined, there are still some deficiencies. Therefore further studies are required.

In conclusion, the expression of miR-34a is associated with bone metastasis and TNM staging, the expression of Ki67 is associated with lymphatic metastasis and TNM staging, and there is a negative correlation between miR-34a and Ki67 expression levels. miR-34a and Ki67 can be used as predictors of NPC-associated mortality. miR-34a, Ki67 and TNM staging are associated with prognosis.

\section{Acknowledgements}

Not applicable.

\section{Funding}

No funding was received.

\section{Availability of data and materials}

The datasets used and/or analyzed during the present study are available from the corresponding author on reasonable request.

\section{Authors' contributions}

LW and CS performed PCR. YZ contributed to the analysis of the observation indexes. CS was responsible for the statistical analysis of the data. LW wrote the manuscript. All authors read and approved the final manuscript.

\section{Ethics approval and consent to participate}

The study was approved by the Ethics Committee of Xiangyang No. 1 People's Hospital (Xiangyang, China). Patients who participated in this research had complete clinical data. Signed informed consents were obtained from the patients and/or guardians.

\section{Patient consent for publication}

Not applicable.

\section{Competing interests}

The authors declare that they have no competing interests.

\section{References}

1. Paul P, Deka H, Malakar AK, Halder B and Chakraborty S: Nasopharyngeal carcinoma: Understanding its molecular biology at a fine scale. Eur J Cancer Prev 27: 33-41, 2018.

2. Fu ZT, Guo XL, Zhang SW, Zeng HM, Sun KX, Chen WQ and He J: Incidence and mortality of nasopharyngeal carcinoma in China, 2014. Zhonghua Zhong Liu Za Zhi 40: 566-571, 2018 (In Chinese).

3. Kong L, Li X, Wang H, He G and Tang A: Calycosin inhibits nasopharyngeal carcinoma cells by influencing EWSAT1 expression to regulate the TRAF6-related pathways. Biomed Pharmacother 106: 342-348, 2018.

4. Huang WB, Chan JYW and Liu DL: Human papillomavirus and World Health Organization type III nasopharyngeal carcinoma: Multicenter study from an endemic area in Southern China. Cancer 124: 530-536, 2018.

5. Fangzheng W, Chuner J, Lei W, Weijun C, Min X, Quanquan S, Tongxin L, Aizawa R, Sakamoto M and Zhenfu F: Outcome and long-term efficacy of four facio-cervical fields conformal radiotherapy for nasopharyngeal carcinoma. Oncotarget 8: 39756-39765, 2017.

6. Wu LR, Liu YT, Jiang N, Fan YX, Wen J, Huang SF, Guo WJ, Bian XH, Wang FJ, Li F, et al: Ten-year survival outcomes for patients with nasopharyngeal carcinoma receiving intensity-modulated radiotherapy: An analysis of 614 patients from a single center. Oral Oncol 69: 26-32, 2017.

7. Zhou Y, Xia L, Lin J, Wang H, Oyang L, Tan S, Tian Y, Su M, Wang $\mathrm{H}$, Cao D, et al: Exosomes in nasopharyngeal carcinoma. J Cancer 9: 767-777, 2018.

8. Witwer KW and Halushka MK: Toward the promise of microRNAs - Enhancing reproducibility and rigor in microRNA research. RNA Biol 13: 1103-1116, 2016.

9. Cheng C, Xiaohua W, Ning J, Dan Z, Chengyun Y, Lijun Z, Li Y, Shengfu H, Hong J and He X: MiR-122 exerts anti-proliferative and apoptotic effects on nasopharyngeal carcinoma cells via the PI3K/AKT signaling pathway. Cell Mol Biol 64: 21-25, 2018.

10. Chen L, Gao H, Liang J, Qiao J, Duan J, Shi H, Zhen T, Li H, Zhang F, Zhu Z, et al: miR-203a-3p promotes colorectal cancer proliferation and migration by targeting PDE4D. Am J Cancer Res 8: 2387-2401, 2018.

11. Li YY, Tao YW, Gao S, Li P, Zheng JM, Zhang SE, Liang J and Zhang Y: Cancer-associated fibroblasts contribute to oral cancer cells proliferation and metastasis via exosome-mediated paracrine miR-34a-5p. EBioMedicine 36: 209-220, 2018.

12. Yang C, Zhang J, Ding M, Xu K, Li L, Mao L and Zheng J: Ki67 targeted strategies for cancer therapy. Clin Transl Oncol 20: $570-575,2018$

13. Zhang J, Liu Y, Deng Y, He J, Lang J and Fan J: Ki67 and nm23 are potential prognostic markers in patients with nasopharyngeal carcinoma. Int J Clin Exp Pathol 9: 6350-6356, 2016.

14. Chen Q, Guo Z, Liu S, Quan P, Cao X, Guo L, Zhang S and Sun $\mathrm{X}$ : The cancer incidence and mortality among children and adolescents during the period of 2010-2014 in Henan Province, China. Cancer Med 8: 814-823, 2019.

15. Livak KJ and Schmittgen TD: Analysis of relative gene expression data using real-time quantitative PCR and the 2(-Delta Delta C(T)) method. Methods 25: 402-408, 2001.

16. Wang J, Wang L, Lou GH, Zeng HR, Hu J, Huang QW, Peng W and Yang XB: Coptidis Rhizoma: A comprehensive review of its traditional uses, botany, phytochemistry, pharmacology and toxicology. Pharm Biol 57: 193-225, 2019.

17. DeBord LC, Pathak RR, Villaneuva M, Liu HC, Harrington DA, Yu W, Lewis MT and Sikora AG: The chick chorioallantoic membrane (CAM) as a versatile patient-derived xenograft (PDX) platform for precision medicine and preclinical research. Am J Cancer Res 8: 1642-1660, 2018.

18. Zhang J, Jia L, Tsang CM and Tsao SW: EBV infection and glucose metabolism in nasopharyngeal carcinoma. Adv Exp Med Biol 1018: 75-90, 2017.

19. Fangzheng W, Chuner J, Quanquan S, Zhimin Y, Tongxin L, Jiping L, Sakamoto M, Peng W, Kaiyuan S, Weifeng Q, et al: Addition of chemotherapy to intensity-modulated radiotherapy does not improve survival in stage II nasopharyngeal carcinoma patients. J Cancer 9: 2030-2037, 2018.

20. Sanchez Calle A, Kawamura Y, Yamamoto Y, Takeshita F and Ochiya T: Emerging roles of long non-coding RNA in cancer. Cancer Sci 109: 2093-2100, 2018. 
21. Huang G, Du MY, Zhu H, Zhang N, Lu ZW, Qian LX, Zhang W, Tian X, He X and Yin L: MiRNA-34a reversed TGF- $\beta$-induced epithelial-mesenchymal transition via suppression of SMAD4 in NPC cells. Biomed Pharmacother 106: 217-224, 2018.

22. Klöppel G and La Rosa S: Ki67 labeling index: Assessment and prognostic role in gastroenteropancreatic neuroendocrine neoplasms. Virchows Arch 472: 341-349, 2018.

23. Shan GP, Wang BB, Zheng P, Du FL and Yang YW: Efficacy and safety of chemotherapy combined with stereotactic radiotherapy in the treatment of nasopharyngeal carcinoma. Med Sci Monit 23: 5630-5636, 2017

24. Guan GF, Zhang DJ, Wen LJ, Yu DJ, Zhao Y, Zhu L, Guo YY and Zheng Y: Prognostic value of TROP2 in human nasopharyngeal carcinoma. Int J Clin Exp Pathol 8: 10995-11004, 2015.
25. Zhao L, Chen H, Hu B, Zhang H and Lin Q: Prognostic significance of Ki67 expression and the derived neutrophil-lymphocyte ratio in nasopharyngeal carcinoma. Cancer Manag Res 10: 1919-1926, 2018

26. Zhao Y, Shen L, Huang X, Jing D, Huang D, Fu J, Li Z, Zhang G and Shen L: High expression of Ki-67 acts a poor prognosis indicator in locally advanced nasopharyngeal carcinoma. Biochem Biophys Res Commun 494: 390-396, 2017.

cc) (i) (8) This work is licensed under a Creative Commons EY AC ND Atribution-NonCommercial-NoDerivatives 4.0 International (CC BY-NC-ND 4.0) License. 